\title{
Characteristic Features of Cognitive, Affective, and Daily Living Functions of Late-Elderly Dementia
}

\author{
Nozomi Hishikawa, ${ }^{1}$ Yusuke Fukui, ${ }^{1}$ Kota Sato, ${ }^{1}$ Syoichiro Kono, ${ }^{1}$ \\ Toru Yamashita, ${ }^{1}$ Yasuyuki Ohta, ${ }^{1}$ Kentaro Deguchi, ${ }^{1}$ and Koji Abe ${ }^{1}$
}

Department of Neurology, Graduate School of Medicine, Dentistry and Pharmaceutical Sciences, Okayama University, Okayama, Japan

Correspondence: Dr. Koji Abe, Department of Neurology, Graduate School of Medicine, Dentistry and Pharmaceutical Sciences, Okayama University, 2-5-1 Shikatacho Kitaku, Okayama 700-8558, Japan.

Tel.: 81-86-235-7365

Fax: 81-86-235-7368

E-mail: nozomi-hishikawa@okayama-u.ac.jp

Running title: The features of late-elderly dementia 


\begin{abstract}
Aims: The world is rapidly aging, and is facing an increase of late-elderly dementia patients. It is important to investigate the characteristic features of late-elderly dementia in the super-aged country. Methods: We examined 1,554 patients with cognitive decline in our clinic, dividing into 3 subgroups according to the age; young- ( $\leq 64$ years old [y.o.]), middle- (65-74 y.o.), and late-elderly (75 y.o. $\leq$ ), and investigated the cognitive, affective, and activities of daily living functions (ADL), especially in late-elderly patients comparing with young- and middle-elderly patients. Results: Among 1,554 patients, Alzheimer's disease (AD) dominated 62\%, and which was age-dependently increased up to $69 \%$ in late-elderly group. Total scores of 4 cognitive tests were significantly worse with aging with specific subscales of orientation, recall, visual retention, word fluency, etc. On the other hand, total scores of the affective tests showed only an increase in apathy scale in the late-elderly group. Each subgroup showed depressive/depression in 63.2$55.2 \%$, and apathy in 44.2-54.8\%. Furthermore, instrumental ADL items significantly deteriorated in the late-elderly group, which statistically correlated with mini-mental state examination (MMSE) score. Conclusions: These results demonstrates that late-elderly group is characterized by significant cognitive declines, increasing apathy, more than half in depressive/depression and apathy, and instrumental ADL decrease. The cognitive decline may be related to such affective and ADL declines.
\end{abstract}

Keywords: affective functions, cognitive function, daily living function, late-elderly dementia, super-aged country 


\section{Introduction}

The world is rapidly aging, where elder population is growing not only in developed countries but also in developing countries. The country is classified as an aged country when elder populations (65 years old [y.o.] or more) occupies more than $10 \%$ of total population, and when more than $20 \%$ a super-aged country. The number of patients with dementia worldwide was currently estimated at 35.6 million in 2010, and this number will increase to 65.7 million by 2030 and more than 115.4 million by $2050 .{ }^{1}$ Japan is one of the super-aged countries in the world, and is facing a rapid increase in number of dementia. Among them, Alzheimer's disease (AD) is the most common, ${ }^{2}$ followed by vascular dementia (VaD), dementia with Lewy bodies (DLB), Parkinson's disease with dementia (PDD), frontotemporal dementia (FTD), idiopathic normal pressure hydrocephalus (iNPH), and so on.

Clinical pictures of dementia have been well documented mainly in presenile or early senile patients. However, there has no such report specifically focusing on the agedependent differences among presenile (young-elderly, < 65 y.o.), early-elderly (65-74 y.o.) and late-elderly (75 y.o. $\leq$ ) patients with dementia. In super-aged country, lateelderly people dominate among aged population (age $\geq 65$ y.o.). In this study, therefore, we examined the ambulatory dementia patients in our clinic, and investigated the cognitive, affective, and activities of daily living functions (ADL) especially in lateelderly dementia comparing with young- and middle-elderly dementia. 


\section{Subjects and methods}

\section{Subjects}

With this retrospective study, we investigated 1,554 ambulatory patients (617 males and 937 females) with cognitive decline at Department of Neurology, Okayama University Hospital. Their medical records of 9,775 patients were reviewed, and the patients were selected who complained of forgetfulness or were suspected to suffer from cognitive decline by their attending doctor. Their average age at the first visit to our hospital was $77.9 \pm 9.6$ y.o. (mean \pm SD) (range 22-104 y.o.). Physical, cognitive, affective, and daily living functions of all patients were measured at first visit to our clinic. We analyzed the patients with dividing into 3 groups according to the age; young- $(\leq 64$ y.o.), middle- (65-74 y.o.), and late-elderly (75 y.o. $\leq$ ).

We have diagnosed as $\mathrm{AD}$ and $\mathrm{VaD}$ according to the National Institute of Neurological and Communicative Disorders Association criteria (NINCDS-ADRDA), ${ }^{3}$ and the National Institute of Neurological Disorders and Stroke and the Association Internationale pour la Recherché et l'Enseignement en Neurosciences (NINDS-AIREN). ${ }^{4}$ Diagnosis of DLB and FTD met each criteria for probable DLB $^{5}$ and frontotemporal lobar degeneration. ${ }^{6}$ Diagnosis of PDD referred to diagnostic criteria of DLB. ${ }^{5}$ Mild cognitive impairment due to $\mathrm{AD}(\mathrm{MCI})$ subjects have been judged based on the $\mathrm{AD}$ neuroimaging initiative (ADNI) criteria, which consists of mini-mental state examination (MMSE) scores $\geqq 24$, a memory complaint, a clinical dementia rating (CDR $)^{7,8}$ of 0.5 , essentially preserved basic ADL, absence of dementia. ${ }^{9}$

This study was approved by the Ethics Committee on Epidemiological Studies of the Okayama University Graduate School of Medicine, Dentistry and Pharmaceutical Sciences (approval number 694). 


\section{Cognitive and affective functions, and activity of daily living (ADL)}

In all patients, we performed neurological examination and cognitive functional tests, including MMSE, Hasegawa dementia score-revised (HDS-R), frontal assessment battery (FAB), and Montreal cognitive assessment (MoCA). Behavioral and psychological symptoms of dementia (BPSD) were evaluated using the Abe's BPSD score (ABS), ${ }^{10}$ neuropsychiatric inventory (NPI), geriatric depression scale (GDS) and apathy scale (AS). ADL of the patients was also assessed using the Alzheimer's Disease Cooperative StudyActivities of Daily Living (ADCS-ADL).

\section{Statistical analysis}

We performed statistical analysis using software SPSS 22.0.0.0 (SPSS Inc., Chicago, IL, USA). We performed Kruskal-Wallis tests to compare each cognitive and affective functional assessments with young-, middle-, and late-elderly dementia patients. p-values less than 0.05 were considered to be significantly different. In addition, trend analyses used the Jonckheere-Terpstra test. $\mathrm{p}<0.05$ was considered to indicate statistical significance

\section{Results}

The demographic data of 1,554 patients with dementia is shown in Fig. 1A-D. We analyzed the patients with dividing into 3 subgroups according to onset of the age. Our patients consisted of 7.2\% young- ( $n=112,60$ males, 52 females), $20.3 \%$ middle- $(n=315$, 141 males, 174 females), and 72.5\% late-elderly ( $n=1127,416$ males, 711 females) 
dementia patients (Fig. 1A). In the groups of young- and middle-elderly dementia there was no significant difference in number of gender, but among late-elderly dementia the ratio of male to female was 1 to 1.7 (Fig. 1B). The most common etiologic groups were AD (62\%), MCI (12\%), VaD (9\%), PDD, FTD and DLB (3\%, respectively) (Fig. 1C). The etiologic data by the 3 divided subgroups showed the proportion of $\mathrm{AD}$ was increased with aging; 30\% in young-elderly group, 51\% in middle-elderly group, and 69\% in lateelderly group. The ratios of MCI and VaD were decreased with aging; each MCI rate was $19 \%, 13 \%$, and $11 \%$, and each $\mathrm{VaD}$ rate was $16 \%, 9 \%$, and $8 \%$, respectively. Among lateelderly dementia, (Fig. 1D, top) the total rate of $\mathrm{AD}$ and MCI was $80 \%$, and the numbers of PDD, DLB, and iNPH patients increased with aging, which were the distinctive features of late-elderly group. On the other hand, the features of young-elderly dementia were relatively high in alcoholic encephalopathy (7\%), dentatorubral-pallidoluysian atrophy (DRPLA) (3\%), Creutzfeldt-Jakob disease (CJD) (2\%), and others including dementia with collagen disease (3\%), encephalitis (3\%), and cerebral autosomal dominant arteriopathy with subcortical infarct and leukoencephalopathy (CADASIL) (2\%) (Fig. 1D, bottom).

Fig. 2 represents the cognitive functions of the 1,554 patients divided in 3 subgroups. Total scores of MMSE, HDS-R, FAB, and MoCA were significantly worse with aging (Fig. 2A). For example, MMSE score of late-elderly dementia (19.4 \pm 5.9$)$ was significantly lower than the young-elderly (21.5 \pm 6.8$)$ and the middle-elderly (20.8 \pm 6.1$)$ groups ( ${ }^{*} \mathrm{p}<0.05$ vs young-elderly and middle-elderly). HDS-R, FAB, and MoCA scores of late-elderly (17.5 $\pm 7.0,10.5 \pm 4.3,15.1 \pm 6.0$, respectively) showed similar decrease than the young-elderly (21.2 $\pm 7.4,12.6 \pm 4.9,12.6 \pm 4.9$, respectively) and the middleelderly (20.1 $\pm 6.8,11.9 \pm 4.4,17.1 \pm 6.3$, respectively) groups $\left({ }^{*} \mathrm{p}<0.05\right.$ vs young-elderly 
and middle-elderly). These 3 subgroups showed a decreasing trend in all 4 scores $\left({ }^{\#} \mathrm{p}<0.01\right)$.

Subscale analyses of these cognitive tests showed a tend to worse with aged-groups within 3 subgroups in the tasks of orientation (MMSE, HDS-R, and MoCA), recall (MMSE, HDS-R, and MoCA), visual retention (HDS-R), word fluency (HDS-R), conceptualization (FAB and MoCA), letter fluency (FAB), conflict (FAB), go/no-go (inhibitory control) (FAB), trail making test (TMT) (MoCA), naming (MoCA), and attention to letter (MoCA) (Fig. 2B, Jonckheere-Tepstra Test $\# p<0.05$, $\# \# p<0.001$ ). On the other hand, direct comparisons among 3 subgroups showed that the late-elderly dementia group declined in the following subscales comparing with the young- and/or middle-elderly groups; in MMSE: the time orientation (young-elderly $3.4 \pm 1.6$ points, middle-elderly $3.3 \pm 1.7$, late-elderly $2.6 \pm 1.7)$, place orientation $(4.1 \pm 1.3$, $4.0 \pm 1.4$, $3.8 \pm 1.1)$, and recall $(1.9 \pm 1.0,1.5 \pm 1.1,1.1 \pm 1.1)\left({ }^{*} \mathrm{p}<0.05\right.$ late-elderly vs youngelderly, middle-elderly, respectively), in HDS-R: time orientation (young-elderly $2.6 \pm$ 1.4, middle-elderly $2.5 \pm 1.5$, late-elderly $2.0 \pm 1.5$ ), three words recall (4.3 $\pm 1.9,3.4 \pm$ 2.0, $3.2 \pm 2.1)$, visual retention ( $3.9 \pm 1.4,3.6 \pm 1.4,3.4 \pm 1.4)$, and word fluency (3.3 \pm 2.1, $3.0 \pm 2.2,2.9 \pm 2.1)\left({ }^{*} \mathrm{p}<0.05\right.$ late-elderly vs young-elderly, middle-elderly, respectively), in FAB: conceptualization (young-elderly $2.4 \pm 0.9$, middle-elderly $2.1 \pm$ 1.1, late-elderly $1.8 \pm 1.1 ;{ }^{*} \mathrm{p}<0.05$ vs young-elderly), conflict $(2.2 \pm 1.2,1.8 \pm 1.3,1.6 \pm$ $1.3 ;{ }^{*} \mathrm{p}<0.05$ vs young-elderly), letter fluency $\left(1.6 \pm 1.2,1.5 \pm 1.1,1.4 \pm 1.0 ;{ }^{*} \mathrm{p}<0.05\right.$ vs young-elderly, middle-elderly, respectively), and go/no-go (1.7 $\pm 1.4,1.4 \pm 1.4,1.0 \pm 1.2$; p<0.05 vs young-elderly, middle-elderly, respectively), and in MoCA: TMT (0.6 \pm 0.5 , $0.3 \pm 0.5,0.2 \pm 0.4 ;{ }^{*} \mathrm{p}<0.05$ vs young-elderly, middle-elderly, respectively), clock drawing ( $2.1 \pm 0.9,2.2 \pm 0.9,1.8 \pm 1.0 ;{ }^{*} \mathrm{p}<0.05$ vs middle-elderly), naming ( $2.6 \pm 0.8$ 
$2.5 \pm 0.9,2.2 \pm 1.0 ;{ }^{*} \mathrm{p}<0.05$ vs young-elderly, middle-elderly, respectively), attention to letter $\left(0.7 \pm 0.5,0.6 \pm 0.5,0.5 \pm 0.5 ;{ }^{*} \mathrm{p}<0.05\right.$ vs young-elderly), conceptualization $(1.7 \pm$ 0.5, $1.5 \pm 0.7,1.4 \pm 0.8 ;{ }^{*} \mathrm{p}<0.05$ vs young-elderly $)$, delayed recall $(1.7 \pm 1.8,1.1 \pm 1.6$ $0.6 \pm 1.2 ;{ }^{*} \mathrm{p}<0.05$ vs young-elderly), and orientation $(4.7 \pm 1.8,4.5 \pm 1.8,4.2 \pm 1.8$; ${ }^{*} \mathrm{p}<0.05$ vs young-elderly, middle-elderly, respectively).

Fig. 3 showed the comparison of affective functions among 3 subgroups. Although no differences among 3 subgroups were found in total scores of GDS, ABS, and NPI, the average GDS scores of all 3 subgroups met depressive state (GDS=5-9 points), i.e., young-elderly group $6.2 \pm 2.1$, middle-elderly group $5.4 \pm 2.2$, and late-elderly group 5.3 \pm 2.0 (Fig. 3A). On the other hand, the average AS score of the late-elderly group (17.5 \pm 2.5 points $)$ was higher $\left({ }^{*} \mathrm{p}<0.05\right)$ than middle-elderly group $(15.1 \pm 2.5)$, while all of their AS scores were higher than the cut-off point ( $\geq 16$ points) of apathy (Fig. 3A). The subscale of each affective score showed significant differences in the happiness of lateelderly patients in GDS subscale ( ${ }^{*} \mathrm{p}<0.05$ vs young-elderly) (Fig. 3B, left), in the future planning and an idea for what to do in AS subscale $\left({ }^{*} \mathrm{p}<0.05\right.$ vs middle-elderly) (Fig. 3B, right), and irritability in the subscale of NPI in the late-elderly group $\left({ }^{*} \mathrm{p}<0.05\right.$ vs youngelderly) (Fig. 3C, right).

Among late-elderly group, 39.2\% represented the depressive state (GDS: 5-9 points), and $16.0 \%$ showed depression (GDS: 10-15 points) (Fig. 4, left). Out of the late-elderly dementia patients, $54.8 \%$ displayed apathy (AS: $16-42$ points), which were $47.4 \%$ in young-elderly and $44.2 \%$ in middle-elderly groups (Fig. 4, right).

In terms of ADL scores among 3 subgroups, the late-elderly group showed a significant decline $\left(18.7 \pm 3.6,{ }^{*} \mathrm{p}<0.05\right)$ than middle-elderly $(20.9 \pm 3.5)$ (Fig. 5A). A statistically weak correlation was observed between MMSE and ADCS-ADL in each 3 
subgroup (young-elderly: $r^{2}=0.3593$, middle-elderly: $r^{2}=0.2766$, late-elderly $r^{2}=0.2985$, respectively) (Fig. 5B). Subscales analyses of ADCS-ADL showed no difference or trend in 10 basic ADL items, but showed a significant difference in 4 instrumental ADL items such as watching TV, mobile phone, internet inspects, and shopping in the late-elderly dementia patients (Fig. 5C).

\section{Discussion}

In this clinic-based cross-sectional study, we examined cognitive, affective, and ADL functions of dementia patients in 3 age-dependent subgroups, i.e., young-, middle-, and late-elderly dementia groups. We found the differences between the 3 subgroups, and disclosed the characteristic features of late-elderly group, which is expected to increase all over the world. This study showed the predominance of late-elderly dementia (72.5\%) (Fig. 1A), the female predominance in late-elderly group (Fig. 1B), ${ }^{11}$ the age-dependent increase of $\mathrm{AD}$ with the largest proportion (69\%) among late-elderly dementia (Fig. 1C and 1D), and age-dependent decrease of MCI from 19 to $11 \%$ and VaD from 16 to $8 \%$ (Fig. 3D).

Total scores of all 4 cognitive functional tests became worse in an age-dependent manner (Fig. 2A), and subscale analyses revealed significant differences in MMSE, HDSR, FAB and MoCA among 3 subgroups (Fig. 2B). Relative to the young- and middleelderly groups, the late-elderly group showed declines in "orientation (MMSE, HDS-R, MoCA)”, “recall (MMSE, HDS-R, MoCA)”, “visual retention (HDS-R)”, “word fluency (HDS-R)”, “conceptualization (FAB, MoCA)”, “letter fluency (FAB)”, “conflict (FAB)”, “inhibitory control (FAB)”, “TMT (MoCA)”, “clock drawing test (MoCA)”, “naming (MoCA)", and "attention to letter (MoCA)" (Fig. 2B), those represent dysfunctions of the 
frontal, parietal and temporal functions in late-elderly subgroup. Previous study reported a decrease in the regional cerebral blood flow (rCBF) in frontoparietal cerebral cortices with aging (31-78 years old) even in the normal subjects. ${ }^{12}$ On the other hand, rCBF of AD patient decreased in the posterior cingulate gyri, precunei, and parietal cortices from very early stage. ${ }^{13}$

Previous studies reported late-elderly AD patients showed a poorer performance on memory and orientation measures than early-elderly $\mathrm{AD}$, but relatively preserved language, concentration, recall, and frontal-executive functions. ${ }^{14,} 15$ However, our present study showed no significant difference among 3 subgroups for concentration (calculation, immediate memory, and digit span), language (letter fluency), and construction (figure copy), but showed significant declines in the delayed recall, orientation (especially to time orientation) and frontal-executive functions in the lateelderly group (Fig. 2B, C). Recent studies showed more than 25\% subjects with pathological diagnosis of AD coexisted with cerebrovascular diseases (CVD), ${ }^{16}$ and agedependent increase of AD plus CVD pathology. ${ }^{2}$ Combined with our present data, more attention should be paid for their frontal dysfunction, especially in the late elderly patients.

Previous studies showed an association of cognitive decline to affective functions, where depression was more popular among young-elderly dementia, ${ }^{17}$ and delusion, hallucination, agitation, disinhibition, and aberrant motor behavior were more frequently observed in late-elderly AD. ${ }^{18}$ The present study showed no significant difference among 3 age-dependent subgroups in GDS, ABS, and NPI, except for AS score (Fig. 3A). However, each subscale analysis revealed that the young-elderly group showed a poorer happiness feeling than the late-elderly group (Fig. 3B, left), and late-elderly group lacked a future planning and an idea for what to do (Fig. 3B, right). The mean score of irritability 
of young-elderly group was higher than that of late-elderly group (Fig. 3C, right). Our present study also showed that more than half of each group were depressive or depression, and about half of them were apathy. Comparing 3 subgroups, the ratio of depressive or depression decreased from 63.2 to 55.2\%, and the apathy increased from 47.4 to $54.8 \%$ with age (Fig. 4). The nationwide hospital-based study in Korea showed that depression (50.6\%) and apathy (49.6\%) were the most common symptoms among mild-to-moderate AD patients. ${ }^{19}$ Psychological symptoms are occasionally affected by social or familiar environment, and BPSD vary by nation, culture, age, gender, and duration of illness. ${ }^{19-23}$

The present study also showed that a significant ADL deterioration especially in the late-elderly group, which statistically correlated with MMSE (Fig. 5A, B). Subscale analyses of ADL revealed 4 instrumental ADL items declined in the late-elderly group without regard to a preserved basic ADL (Fig. 5C), which might be related not to have such customs of using these instruments in elderly person, or which could suggest the parietal and frontal cerebral cortices were mainly affected in these late-elderly dementia.

Our study showed the complete picture of the ambulatory patients with cognitive decline to investigate the characteristic features of late-elderly dementia in the super-aged country. Constituent of dementia patients are different among the three groups according to the age. For instance, there is a significant difference in the prevalence of AD. So our results were influenced by the type of dementia.

In summary, the present study demonstrates that late-elderly dementia group showed significant cognitive declines especially in the delayed recall, orientation and frontalexecutive functions. More than half of dementia patients were depressive or depression, and $54.8 \%$ of the late-elderly group were apathy. Late-elderly dementia patients showed 
declines in instrumental ADL, relating to cognitive decline. Paying more attention on therapy may be focused on parietal and frontal functions in these late-elderly dementia patients.

\section{Acknowledgements}

This work was partly supported by a Grant-in-Aid for Scientific Research (B) 21390267, (C) 24591263 and Challenging Research 24659651, and by Grants-in-Aid from the Research Committees (Mizusawa H, Nakano I, Nishizawa M, Sasaki H, and Aoki M) from the Ministry of Health, Labour and Welfare of Japan.

\section{Disclosure statement}

The authors declare no conflicts of interest.

\section{References}

1. World Health Organization and Alzheimer's Disease International. Dementia: A Public Health Priority. Geneva: World Health Organization; 2012; 92-93. http://www.who.int/mental_health/publications/dementia_report_2012/en/

2. Jellinger KA, Attems J. Prevalence of dementia disorders in the oldest-old: an autopsy study. Acta Neuropathol. 2010; 119: 421-433.

3. McKhann G, Drachman D, Folstein M, Katzman R, Price D, Stadlan EM. Clinical diagnosis of Alzheimer's disease: report of the NINCDS-ADRDA Work Group under the auspices of Department of Health and Human Services Task Force on Alzheimer's Disease. Neurology. 1984; 34: 939-944. 
4. Román GC, Tatemichi TK, Erkinjuntti T, et al. Vascular dementia: diagnostic criteria for research studies. Report of the NINDS-AIREN International Workshop. Neurology. 1993; 43: 250-260.

5. McKeith IG, Dickson DW, Lowe J, et al. Consortium on DLB. Diagnosis and management of dementia with Lewy bodies: third report of the DLB Consortium. Neurology. 2005; 65: 1863-1872.

6. Neary D, Snowden JS, Gustafson L, et al. Frontotemporal lobar degeneration: a consensus on clinical diagnostic criteria. Neurology. 1998; 51: 1546-1554.

7. Morris JC. The Clinical Dementia Rating (CDR): current version and scoring rules. Neurology. 1993; 43: 2412-2414.

8. Mueller SG, Weiner MW, Thal LJ, et al. Ways toward an early diagnosis in Alzheimer's disease: the Alzheimer's Disease Neuroimaging Initiative (ADNI). Alzheimers Dement. 2005;1: 55-66.

9. Petersen RC, Roberts RO, Knopman DS, et al. Mild cognitive impairment: ten years later. Arch Neurol. 2009; 66: 1447-1455.

10. Abe K, Kurata T, Deguchi K, et al. A new simple score (ABS) for screening behavioral and psychological symptoms of dementia. 21th European Congress of Psychiatry (EPA2013) in Nice, France.

11. Launer LJ, Andersen K, Dewey ME, et al. Rates and risk factors for dementia and Alzheimer's disease: results from EURODEM pooled analyses. EURODEM Incidence Research Group and Work Groups. European Studies of Dementia. 1999; 52: 78-84.

12. Pagani M, Högberg G, Salmaso D, et al. Regional cerebral blood flow during auditory recall in 47 subjects exposed to assaultive and non-assaultive trauma and developing or not posttraumatic stress disorder. Eur Arch Psychiatry Clin Neurosci. 2005; 255 : 359-65.

13. Johnson KA, Jones K, Holman BL, et al. Preclinical prediction of Alzheimer's disease using SPECT. Neurology. 1998; 50: 1563-1571. 
14. Frisoni GB, Pievani M, Testa C, et al. The topography of grey matter involvement in early and late onset Alzheimer's disease. Brain. 2007; 130: 720-730.

15. Koss E, Edland S, Fillenbaum G, et al. Clinical and neuropsychological differences between patients with earlier and later onset of Alzheimer's disease: A CERAD analysis, Part XII. Neurology. 1996; 46: 136-141.

16. Nagy Z, Esiri MM, Jobst KA, et al. The effects of additional pathology on the cognitive deficit in Alzheimer disease. J Neuropathol Exp Neurol. 1997; 56: 165-170.

17. van Vliet D, de Vugt ME, Köhler S, et al. Awareness and its association with affective symptoms in young-onset and late-onset Alzheimer disease: a prospective study. Alzheimer Dis Assoc Disord. 2013; 27: 265-271.

18. Toyota Y, Ikeda M, Shinagawa S, et al. Comparison of behavioral and psychological symptoms in early-onset and late-onset Alzheimer's disease. Int J Geriatr Psychiatry. 2007; 22: 896-901.

19. Park HK, Na DL, Han SH, et al. Clinical characteristics of a nationwide hospitalbased registry of mild-to-moderate Alzheimer's disease patients in Korea: a CREDOS (Clinical Research Center for Dementia of South Korea) study. J Korean Med Sci. 2011; 26: 1219-1226.

20. Fernández Martínez M, Castro Flores J, Pérez de las Heras S, Mandaluniz Lekumberri A, Gordejuela Menocal M, Zarranz Imirizaldu JJ. Prevalence of neuropsychiatric symptoms in elderly patients with dementia in Mungialde County (Basque Country, Spain). Dement Geriatr Cogn Disord. 2008; 25: 103-108.

21. Hirono N, Mori E, Yasuda M, et al. Factors associated with psychotic symptoms in Alzheimer's disease. J Neurol Neurosurg Psychiatry. 1998; 64: 648-652.

22. Ikeda M, Fukuhara R, Shigenobu K, et al. Dementia associated mental and behavioural disturbances in elderly people in the community: findings from the first Nakayama study. J Neurol Neurosurg Psychiatry. 2004; 75: 146-148.

23. Lyketsos CG, Lopez O, Jones B, Fitzpatrick AL, Breitner J, DeKosky S. Prevalence of neuropsychiatric symptoms in dementia and mild cognitive impairment: results from the cardiovascular health study. JAMA. 2002; 288: 1475-1483. 


\section{Figure legends}

Figure 1. Demographic data of 1,554 patients with dementia. Our patients consisted of 7.2\% young-, 20.3\% middle-, and 72.5\% late-elderly dementia patients (A). Patients of each group were divided by gender (B). Etiologic data of 1,554 dementia patients in our sturdy (C), and their age-dependent 3 subgroups (D).

Figure 2. Cognitive functions in the 1,554 patients divided in 3 age-dependent subgroups. White, gray, and black bars represent young-, middle-, and late-elderly dementia groups, respectively (panels A and B). Panel B shows cognitive subscales as mean percentage, showing significant declines of some subscales in late-elderly group than the young- and the middle-elderly groups $\left({ }^{*} \mathrm{p}<0.05,{ }^{* *} \mathrm{p}<0.001\right)$ and age-dependent decreasing trends $\left({ }^{\#} \mathrm{p}<0.05,{ }^{\#} \mathrm{p}<0.001\right)$.

Figure 3. Affective functions in the 1,554 patients divided in 3 age-dependent subgroups. White, gray, and black bars representing young-, middle-, and late-elderly dementia groups, respectively, showing a significant increase of AS in the late-elderly group $\left({ }^{*} \mathrm{p}<0.05\right.$ vs middle-elderly group), and some subscales in GDS, AS, and NPI (B) $\left({ }^{*} \mathrm{p}<0.05\right)$.

Figure 4. Prevalence of affective dysfunction in GDS and AS in the 1,554 dementia patients divided in 3 age-dependent subgroups, showing more than a half but agedependent decrease in depressive/depression (left), and more than a half in apathy (right) in late-elderly patients. 
Figure 5. ADCS-ADL scores among 3 subgroups (A), showing a significant decline the late-elderly group than middle-elderly group $\left({ }^{*} \mathrm{p}<0.05\right)$, a statistical correlation between MMSE and ADCS-ADL in each 3 subgroup (young: $r^{2}=0.3593$, middle-elderly: $r^{2}=$ 0.2766, late-elderly $\left.\mathrm{r}^{2}=0.2985\right)(\mathrm{B})$, and a significant decline in 4 instrumental ADL subscale items $\left({ }^{*} \mathrm{p}<0.05\right)(\mathrm{C})$. White, gray, and black bars and symbols represent young-, middle-, and late-elderly dementia groups, respectively. 


\section{Figure 1}

A

\begin{tabular}{|l|l|l|}
\hline $7.2 \%$ & $20.3 \%$ & $72.5 \%$ \\
\hline
\end{tabular}

B

(Number)

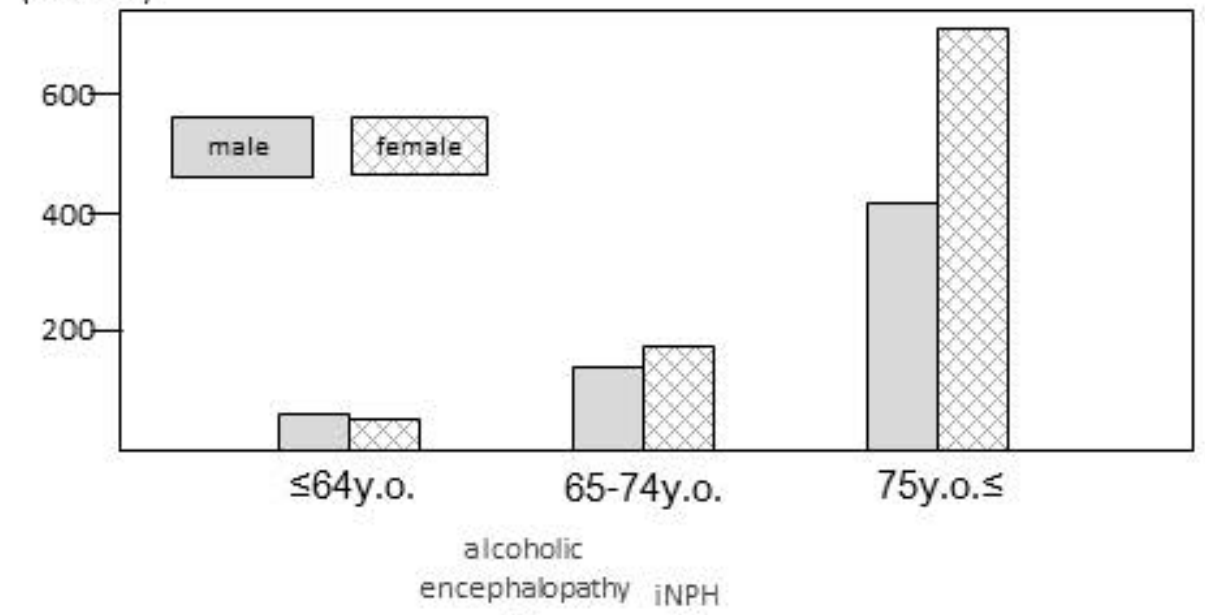

C

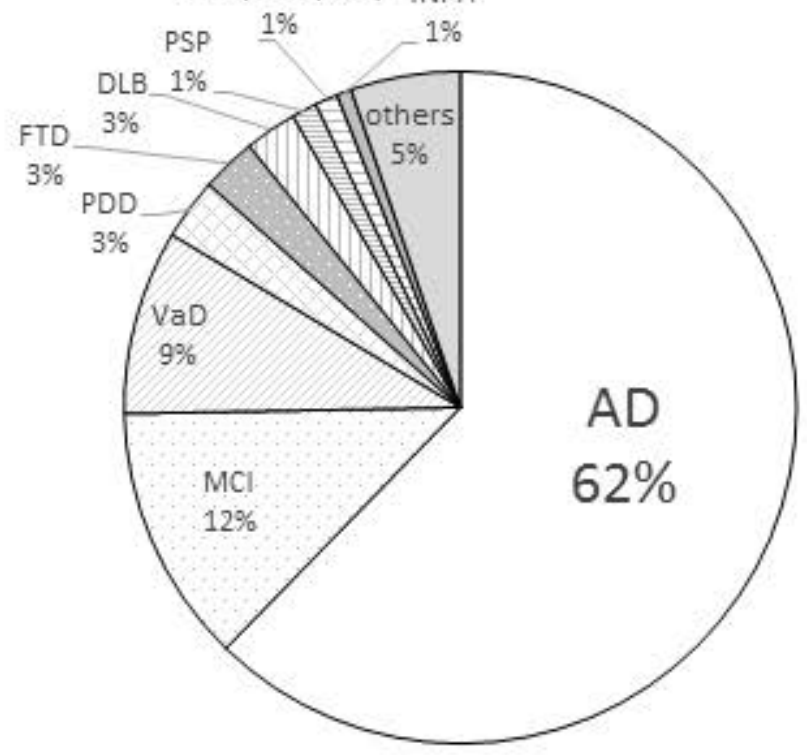

D

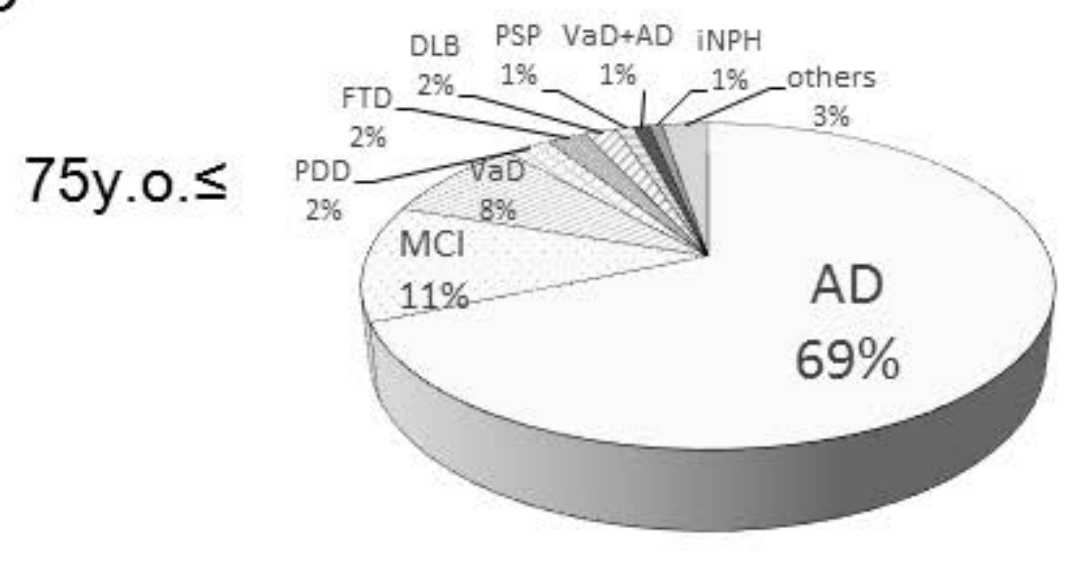

alcoholic
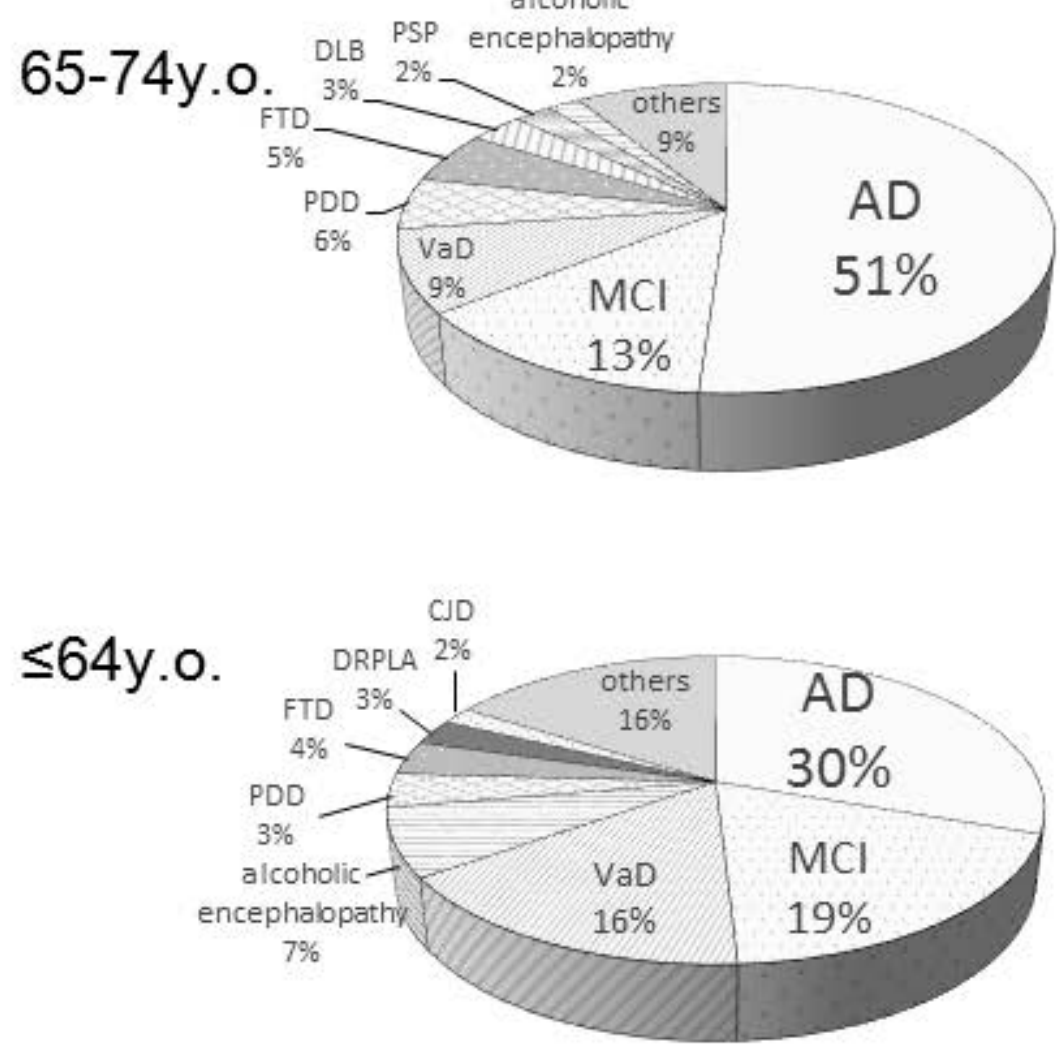
Figure 3

A

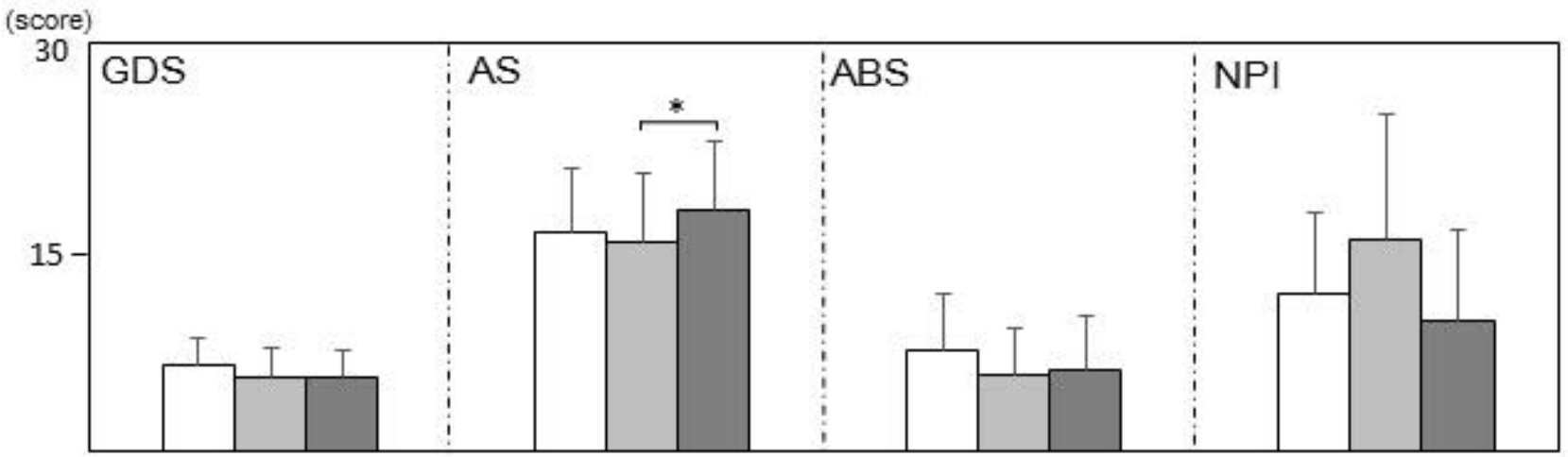

B

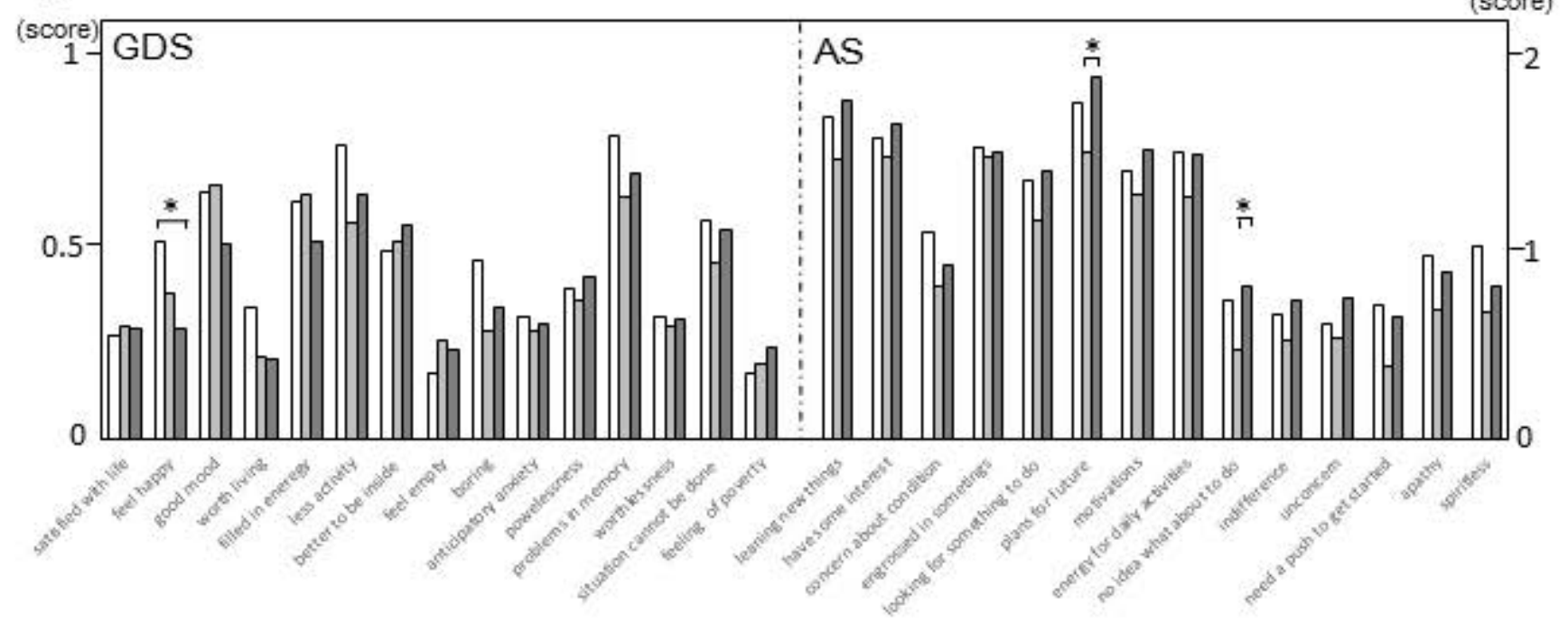

C

(\%) ABS
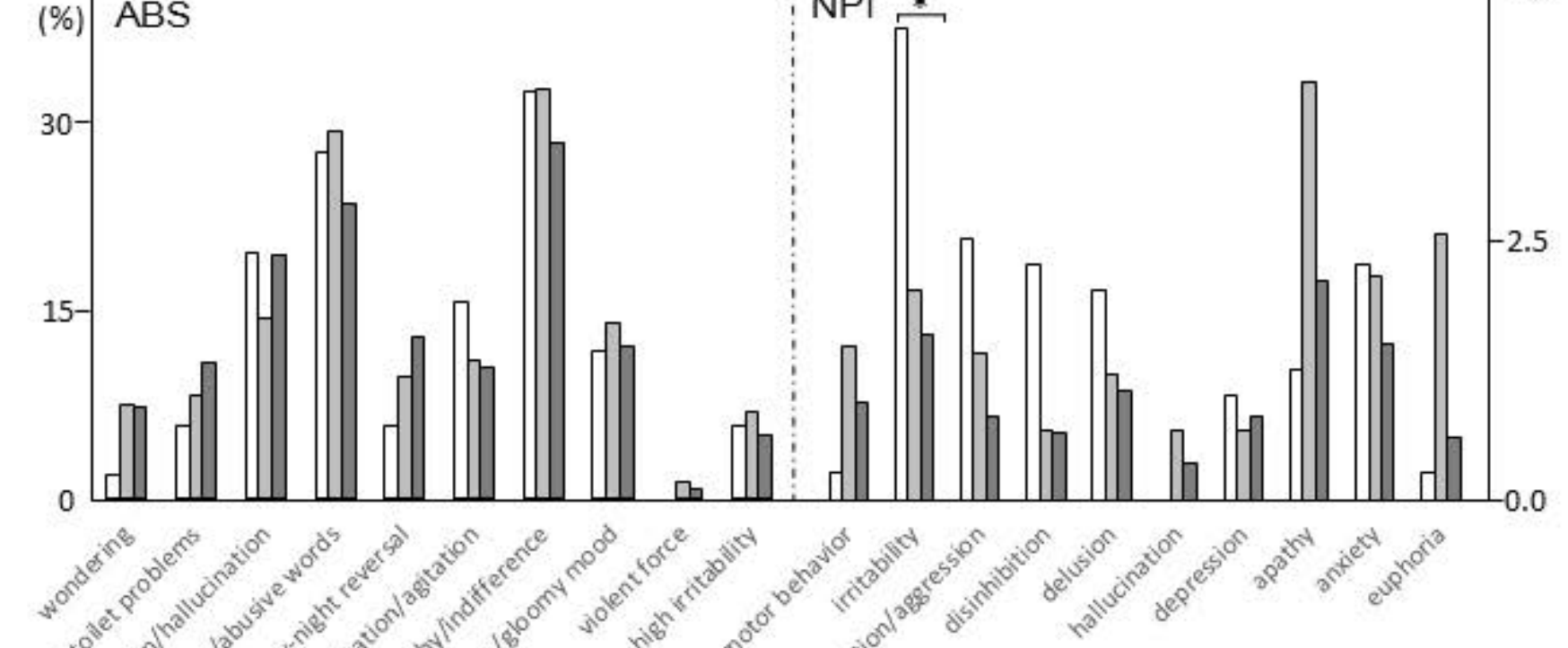
Figure 2

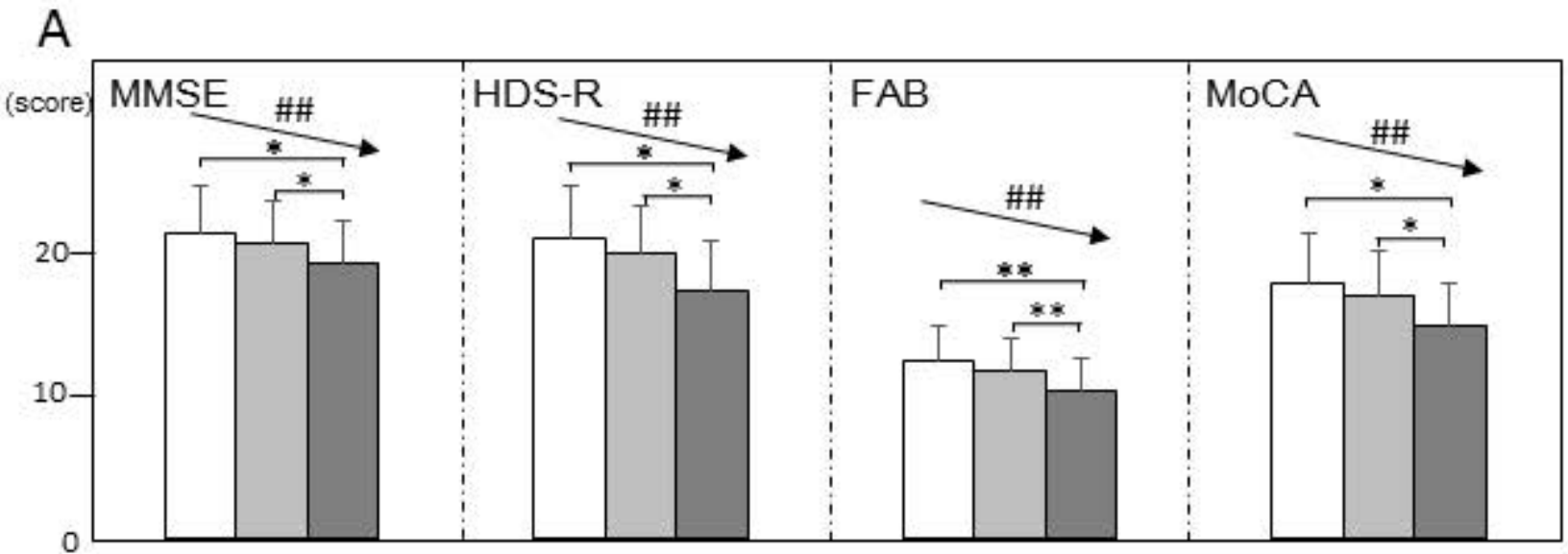

\section{B}
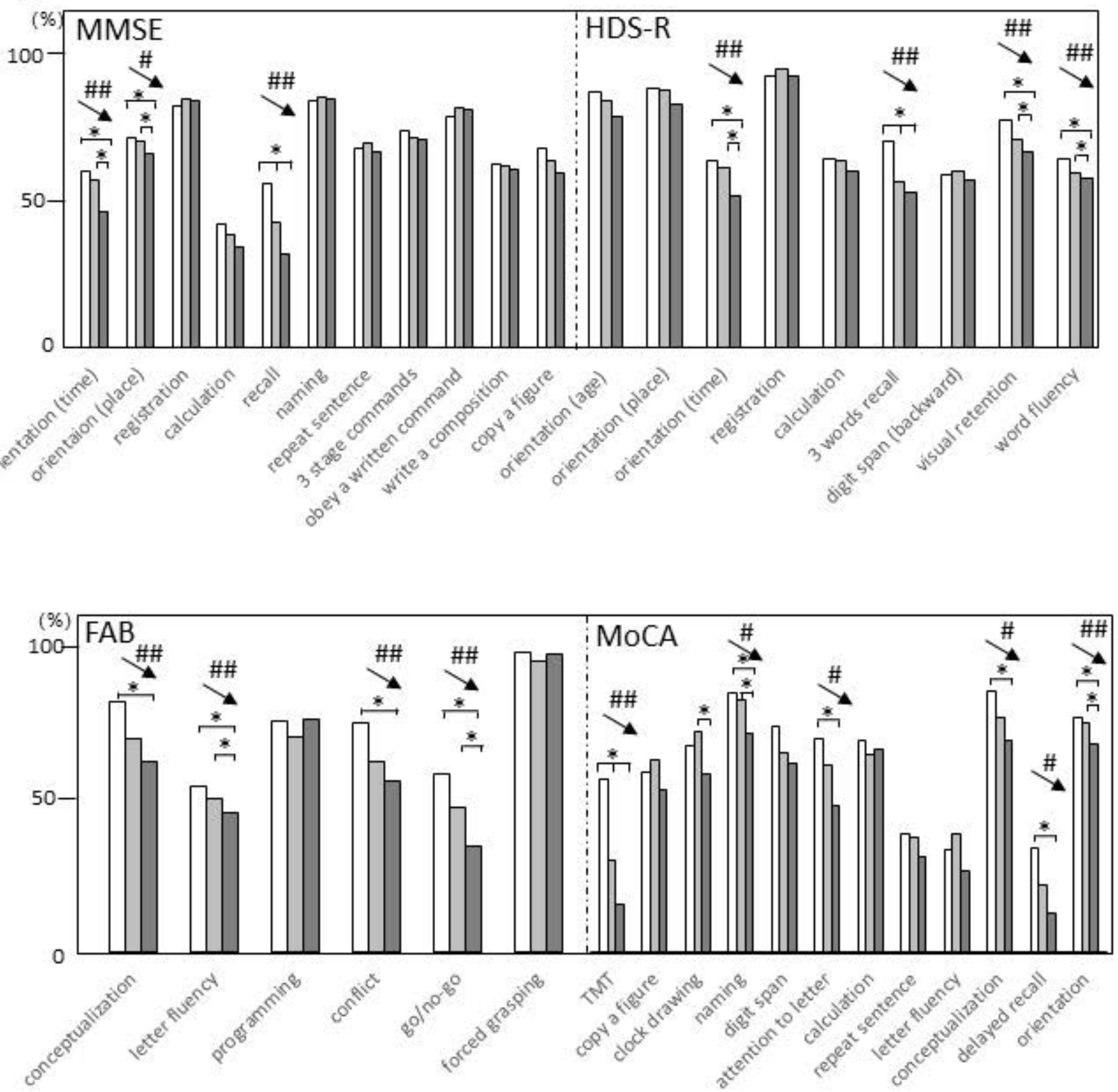
Figure 5

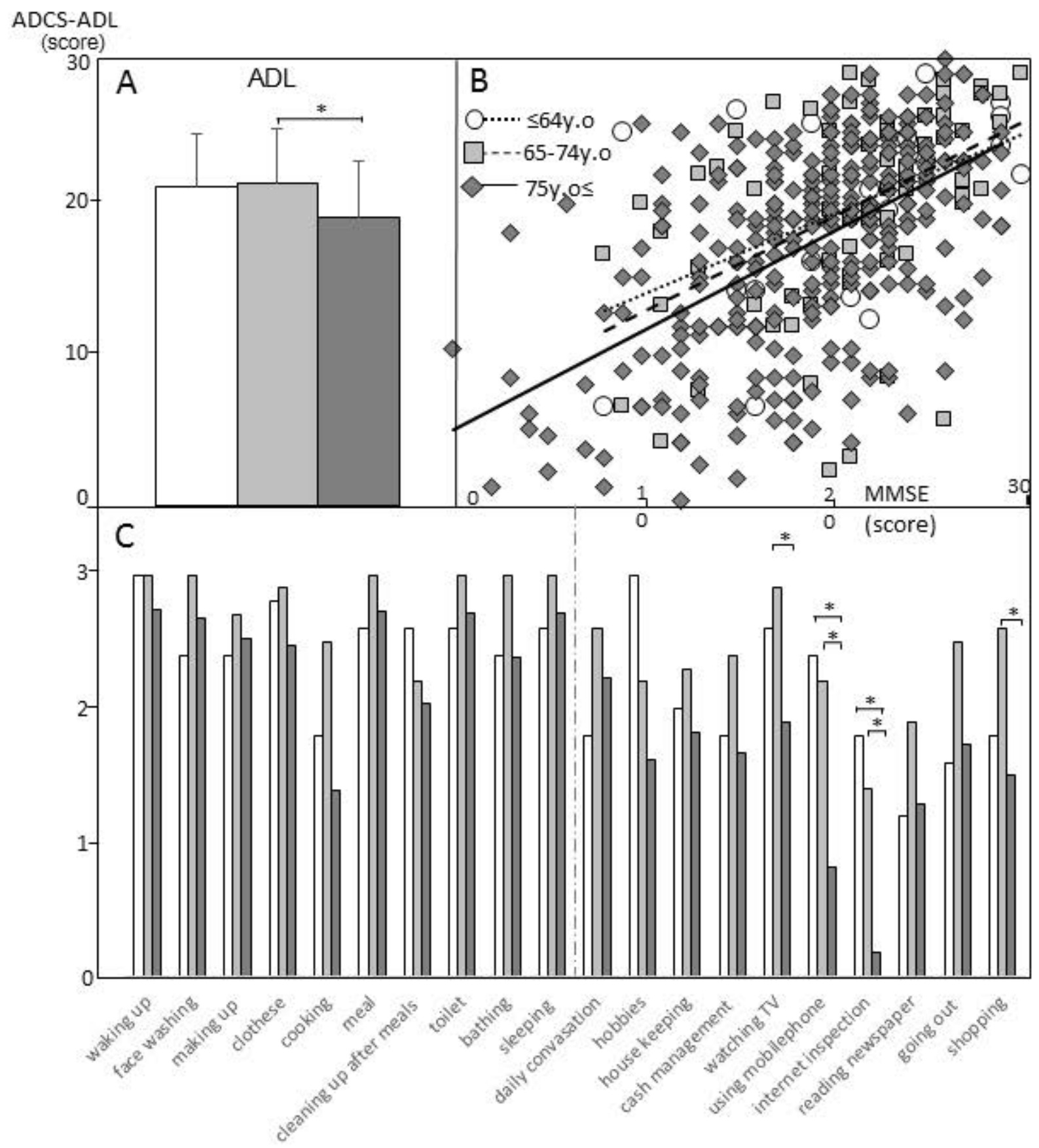


GDS

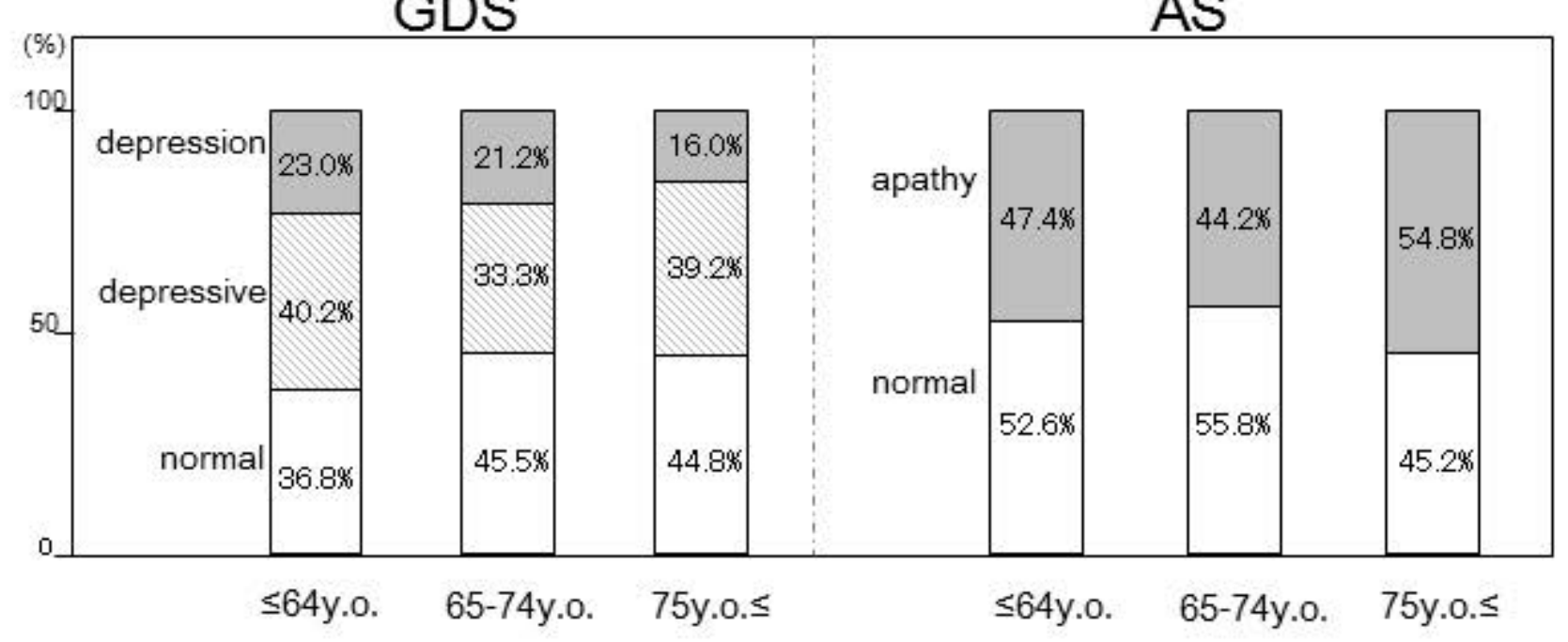

AS 